\title{
Évaluer des compétences : une articulation cubique
}

\section{Competency assessment: A cubic articulation}

\section{Evaluar habilidades: una articulación cúbica}

https://doi.org/10.52358/mm.vi9.244

Nadine Talbot, professeure

Université du Québec à Trois-Rivières, Canada

nadine.talbot@ugtr.ca

Christophe Gremion, maître d'enseignement

Haute école fédérale en formation professionnelle (HEFP), Suisse

christophe.gremion@hefp.swiss

\section{RÉSUMÉ}

Depuis la mise en place de programmes centrés sur le développement des compétences, l'évaluation des apprentissages fait l'objet de nombreux questionnements de la part des acteurs des milieux de formation. Les questionnements se sont accentués depuis le virage obligé vers l'enseignement à distance en raison du contexte pandémique. Plusieurs enseignants témoignent de préoccupations grandissantes. Comment évaluer lorsque les élèves ne sont pas là? Comment vérifier qu'ils ne trichent pas et que ce sont bien leurs réponses qu'ils retournent? Le risque du plagiat augmente avec la distance alors que les évaluations constituées d'items à choix multiples (QCM) ou d'items à réponse courte sont favorisées par les outils numériques. Bien qu'ils facilitent les rétroactions en période d'enseignement à distance et diminuent le temps de correction, ces types d'items ne permettent pas d'évaluer tous les objets d'apprentissage visés. C'est bien là que le bât blesse. Évaluer des compétences ne se fera pas par QCM. II devient essentiel de revenir à l'essentiel, soit comment évaluer les compétences, et ce, malgré la distance. Cet article propose une réflexion sur l'évaluation scolaire et présente un modèle de questionnements des visées de l'évaluation à distance. 
Mots-clés : évaluation des compétences, enseignement présentiel, enseignement à distance, alignement pédagogique

\section{ABSTRACT}

Since the implementation of programs focus on competencies development, the learning assessment has been the subject of many questions on the part of the actors of the education context. Due to the pandemic context, questions have increased on the shift towards distance teaching. Several teachers testify to growing concerns. How to assess when the students are not there? How can the teacher ensure studentsare not cheating? The risk of plagiarism increases in distance learning settings, especially when digitals tools favour assessments consisting of multiple-choice questions (MCQ) or short-answer items. Although they facilitate feedback during distance teaching and decrease correction time, are these toolsinteresting alternative if they do not allow the assessment of all the learning objects targeted? This is where the bottom hurts. MCQ do not assess competencies. It becomes essential to get back to basics, which is how to assess skills, despite the distance. This article offers a reflection on academic assessment and presents a questioning model of the aims of distance assessment.

Keywords: competency assessment, face-to-face teaching, distance teaching, constructive alignment

\section{RESUMEN}

Desde la implementación de programas enfocados al desarrollo de habilidades, la evaluación de aprendizajes ha sido objeto de múltiples interrogantes por parte de quienes trabajan en entornos de formación. Las preguntas han aumentado desde el cambio hacia la educación a distancia debido al contexto de la pandemia. Varios maestros dan testimonio de preocupaciones crecientes. ¿Cómo evaluar cuando los estudiantes no están allí? ¿Cómo se comprueba que no están haciendo trampa y que son sus respuestas las que están devolviendo? El riesgo de plagio aumenta con la distancia, ya que las herramientas digitales favorecen las evaluaciones que consisten en elementos de opción múltiple o respuestas cortas. Aunque facilitan la retroalimentación durante la educación a distancia y reducen el tiempo de corrección, este tipo de elementos no evalúa todos los objetos de aprendizaje involucrados. Aquí es donde el zapato aprieta. Las preguntas de opción múltiple no evaluarán las habilidades. Se vuelve fundamental volver a lo básico, que es cómo evaluar las habilidades, a pesar de la distancia. Este artículo ofrece una reflexión sobre la evaluación escolar y presenta un modelo para cuestionar los objetivos de la evaluación a distancia.

Palabras clave: evaluación de habilidades, enseñanza presencial, aprendizaje a distancia, alineación pedagógica 


\section{Introduction}

De plus en plus de programmes de formation, dans divers pays et différentes disciplines, sont centrés sur le développement des compétences, que ce soit à l'enseignement primaire, secondaire, collégial ou universitaire. À la suite de l'implantation de ces programmes de formation, l'évaluation des apprentissages fait l'objet de nombreux questionnements de la part des différents acteurs des milieux de formation, tout particulièrement les enseignants et les conseillers pédagogiques. Certains se questionnent sur ce qu'implique l'évaluation d'une compétence comparativement à l'évaluation de l'atteinte d'objectifs d'apprentissage. Pour d'autres, la conviction d'une notation en pourcentage semble demeurer. Les questionnements se sont accentués depuis le virage obligé vers l'enseignement à distance à cause du contexte pandémique. Dans ce contexte d'enseignement, les enseignants semblent dépourvus de moyens pour évaluer les compétences de leurs élèves. Plusieurs se rabattent sur l'évaluation par questionnaires en ligne constitués d'items à choix multiples pour lesquels la correction peut être automatisée. Plusieurs enseignants témoignent de la préoccupation grandissante du plagiat alors que les évaluations, même Iorsqu'il s'agit de compétences, sont fréquemment constituées d'items à choix multiples ou d'items à réponse courte afin d'en faciliter la correction. Bien que ces types de questions puissent être particulièrement utiles en contexte d'enseignement à distance, ils ne permettent pas d'évaluer tous les objets d'apprentissage. Il devient donc essentiel, en ces temps de changement, de revenir à l'essentiel : comment évaluer réellement ce qui doit être enseigné?

\section{Des pédagogies dépassées au regard des maux d'aujourd'hui?}

Bien que le développement de compétences soit la finalité de nombre de programmes de formation, plusieurs enseignants témoignent ne pas savoir comment faire pour évaluer le niveau de compétences atteint par un élève. D'ailleurs, il semble y avoir une recrudescence d'évaluations des apprentissages à l'aide d'items à choix multiples ou d'items à réponse courte, laissant de côté l'évaluation en situation ou à l'aide de tâches complexes, encore plus en contexte d'enseignement à distance (Gremion et Leroux, 2019). Lors de ce type d'évaluation, plusieurs enseignants s'étonnent de l'augmentation exponentielle du plagiat constaté lors de telles évaluations, allant jusqu'à se demander si l'évaluation des apprentissages en contexte d'enseignement à distance est possible (Detroz et al., 2020). Afin de contrer le plagiat, certains enseignants privilégient la surveillance à distance par des systèmes comme la reconnaissance faciale de l'élève, pour ne citer que celui-ci. Ces pratiques évaluatives, bien ancrées selon Scallon (2004a), laissent présager un retour au courant encyclopédique, apparu avec Pantagruel à la Renaissance et qui a prévalu jusqu'en 1980 au Québec comme ailleurs (Durand et Chouinard, 2012). À l'époque, les items à choix multiples, les items à réponse vrai ou faux ainsi que les items à réponse courte étaient privilégiés pour évaluer le niveau de connaissances accumulées par l'élève, ce qui était bien le but des programmes de formation en vigueur à cette époque. D'ailleurs, Scallon (2004a, p. 19) précise que « la pratique des examens objectifs s'applique à des objectifs d'apprentissage reliés à des savoirs et des savoir-faire de base, cette méthodologie est dépassée pour inférer des compétences ॥. II semble qu'avec la mise en place de l'approche par compétences, les plans de formation ont changé, souvent bien plus rapidement que les pratiques évaluatives. 


\section{Intention et outils, ou comment ne pas mettre la charrue avant les bœufs}

Un problème émerge de ces constats quant à la pertinence des évaluations et, par extension, celle du jugement évaluatif. En effet, alors que les programmes de formation visent le développement de compétences, les modalités d'évaluation utilisées par certains enseignants n'évaluent que l'accumulation des connaissances acquises par l'élève alors que « le recours à une note de passage comme repère dans le jugement à porter ne tient plus la route » (Scallon, 2004a, p. 15). Si l'alignement pédagogique - le fil conducteur entre ce qui doit être enseigné (ce qui est prévu au programme), ce qui est enseigné et ce qui est évalué - est inexistant, la pertinence de l'évaluation et de ses résultats est alors compromise (Biggs et Tang, 2011). Déjà en 1949, Tyler insistait sur l'importance de la cohérence entre les objectifs d'enseignement, les activités d'apprentissage et l'évaluation des apprentissages. En 1987, Cohen précise que les résultats des élèves sont considérablement plus élevés lorsque l'alignement pédagogique est respecté. Dans cet esprit d'alignement pédagogique, Pellegrino (2012) insiste sur l'importance pour l'enseignant de déterminer, en premier, ce qu'il souhaite évaluer et par la suite de choisir la modalité d'évaluation la plus appropriée. Rieunier (2007) propose aussi de penser et de préparer son évaluation avant même de concevoir le dispositif d'enseignement-apprentissage. Pellegrino (2012) insiste aussi sur l'importance de la pertinence des choix évaluatifs, puisqu'une même modalité d'évaluation ne permet pas d'évaluer tout objet d'apprentissage. Ainsi, une modalité d'évaluation non adaptée à l'objet d'apprentissage ciblé, donc non aligné pédagogiquement, mènera à des conclusions erronées sur les apprentissages réels de l'élève (Pellegrino, 2012). Que se passe-t-il lorsque l'on se doit de soutenir le développement de compétences, mais que l'on utilise des outils peu adaptés pour les évaluer? Qui monterait dans un avion dont les apprentissages du pilote ont été évalués par des questions à choix multiples au lieu de tâches complexes simulant un vol et des vols réels? Qui accepterait de subir une chirurgie par un médecin dont on aurait vérifié les compétences en analysant son temps de connexion au système de formation par des learning analytics?

\section{Compétences, situation et complexité : un triptyque indissociable}

Déjà en 1994, Finson se positionnait sur les modalités d'évaluation dites traditionnelles, tels les items à choix multiples, en affirmant qu'elles ne permettent pas d'évaluer la capacité à résoudre des problèmes ni de réaliser une expérience de laboratoire. II suggère plutôt une évaluation des apprentissages à l'aide de performances, soit de tâches complexes. Ainsi, lors de l'évaluation des acquis dans un contexte d'évaluation de compétences, l'enseignant amène l'élève à mobiliser des connaissances au lieu de lui demander de répondre à des questions nécessitant une réponse déjà connue textuellement. Dans ce contexte de recherche d'évaluation authentique, Scallon (2004b) met l'accent sur la réalisation d'une tâche complexe signifiante pour l'élève lui permettant de mobiliser plusieurs ressources dans le but de démontrer sa compétence. Gérard (2009) ajoute l'importance d'une solution originale et efficace de la part de l'élève. Une tâche complexe est authentique, signifiante pour l'élève, elle ne le guide pas vers la solution, notamment en laissant l'élève discriminer les informations pertinentes et non pertinentes, elle permet à l'élève de mobiliser plusieurs connaissances, habiletés et stratégies, et elle permet plusieurs solutions. La réalisation de plusieurs tâches complexes par l'élève permettra à l'enseignant d'inférer le niveau de compétence développé. Par conséquent, il est à privilégier que toutes les situations d'évaluations réalisées dans le but de porter un jugement consigné au bulletin soient une tâche complexe. Lors de la correction, la démarche peut revêtir plus d'importance que la réponse elle-même. Le jugement transmis par le bulletin 
est alors holistique, puisqu'il fait état de la compétence de l'élève tant sur la démarche que sur la solution proposée, dépassant ainsi la simple somme de notations en pourcentage.

\section{L'évaluation, le but ou le gouvernail, voire le moteur}

En contexte de développement de compétences, l'évaluation se doit aussi d'être intégrée au processus enseignement - apprentissage. Cette volonté se réalise en classe par l'évaluation au service de l'apprentissage et par l'évaluation pour l'apprentissage dans le but d'amener l'élève à s'autoréguler (Vial, 2012). Par l'évaluation au service des apprentissages, l'élève s'autoévalue ou évalue ses pairs afin de déterminer les défis rencontrés lors de la réalisation de tâches complexes, permettant ainsi de déterminer où il se situe quant aux attentes de développement des compétences évaluées (Earl, 2013). Selon Baxter et Shavelson (1994), l'élève devient plus responsable de la progression de ses apprentissages et est impliqué dans leur évaluation en s'autoévaluant, en se fixant des objectifs en lien avec ceux du programme et en discutant avec d'autres élèves. Lors d'une évaluation pour l'apprentissage, l'enseignant donne de la rétroaction à l'élève à la suite de la réalisation d'une tâche complexe pour laquelle il s'est autoévalué ou non. Pour Vial (2012), l'évaluation centrée sur la progression de l'élève est formatrice, puisqu'elle favorise l'appropriation des contenus par la compréhension des critères d'évaluation pour une tâche complexe. Évolution de l'évaluation formative, parfois vue comme synonyme de rétroaction, l'évaluation formatrice (Nunziati, 1990), l'évaluation au service de l'apprentissage et l'évaluation pour l'apprentissage permettent à l'élève de s'orienter dans ses apprentissages, lui offrent l'occasion de s'autoévaluer et de se rendre compte par lui-même du chemin qu'il lui reste à parcourir pour atteindre la compétence visée.

\section{En évaluant à distance, l'objet d'évaluation reste-t-il le même?}

En découle un questionnement sur la possibilité ou non d'évaluer les compétences à distance. La figure 1, adaptée de Gremion et Leroux (2019), propose un cube présentant l'articulation entre les visées de l'évaluation des apprentissages, le contexte d'enseignement ainsi que le type de modalités d'évaluation des apprentissages. L'ensemble des possibilités présentées sont autant de contextes d'évaluation parmi lesquels l'enseignant doit situer la modalité d'évaluation des apprentissages qu'il propose à ses élèves selon les conditions et les intentions d'enseignement.

Cette articulation suppose que l'évaluation des apprentissages, peu importe la discipline d'enseignement, devrait être envisagée selon trois axes. Le premier axe, " évaluer quoi », présente les objets d'évaluation possibles, soit le continuum du degré de complexité des apprentissages visés correspondant au continuum des connaissances vers des compétences nécessitant un haut niveau de mobilisation, qui peuvent aussi, et traditionnellement, être nommés savoirs, savoir-faire, savoir-être et compétences (ou savoir-y-faire). Selon les visées de son programme de formation, l'enseignant évalue l'un ou l'autre de ces objets d'apprentissage. 


\section{Figure 1}

Articulation de l'évaluation des apprentissages

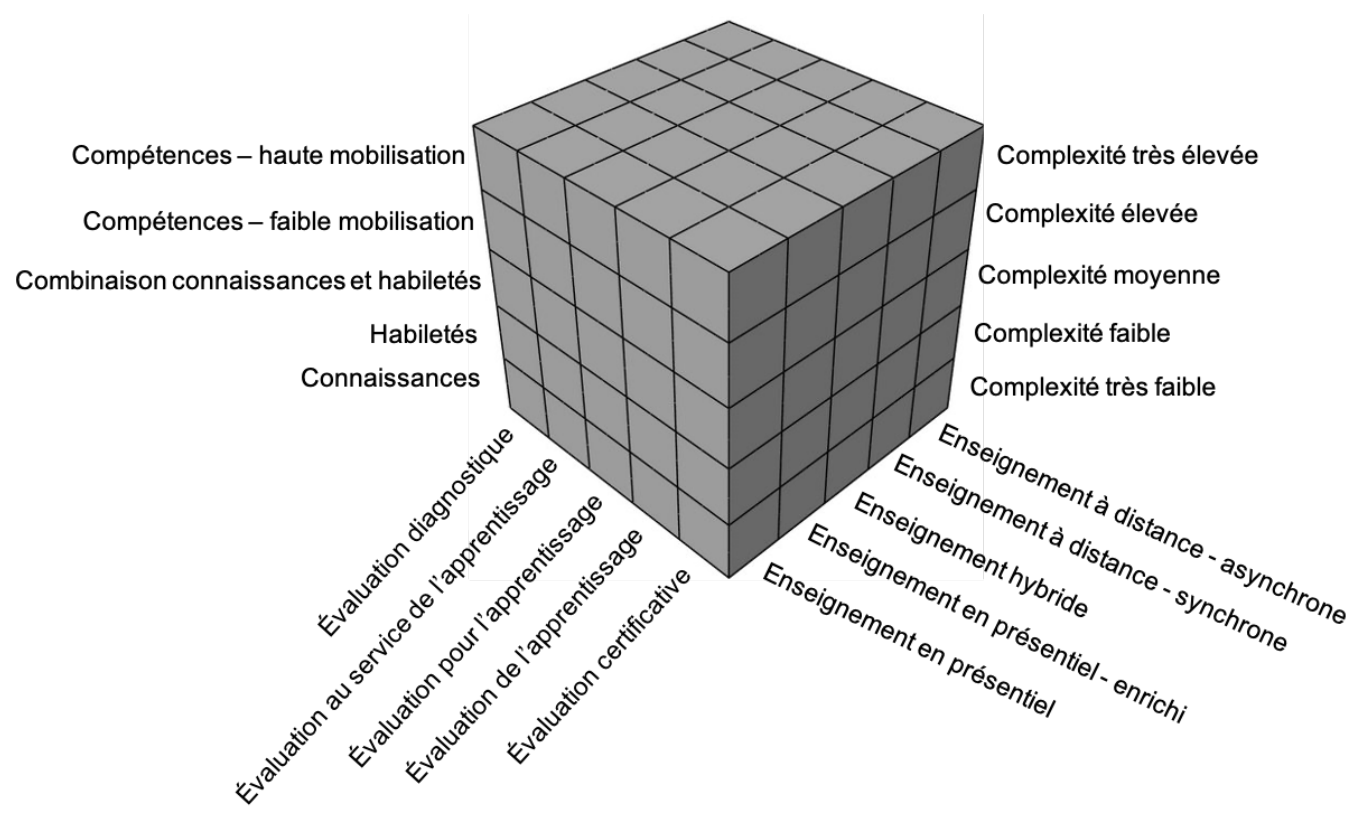

Note. Adapté de Gremion et Leroux (2019); figure du cube Cao et Qin (2015).

Le deuxième axe, « évaluer dans quelle visée », représente chacune des fonctions (visées) de l'évaluation des apprentissages, dans une posture où la régulation des apprentissages par l'élève est centrale, de l'évaluation diagnostique à l'évaluation certificative. Le troisième axe, " évaluer dans quel contexte d'enseignement », présente un ensemble de possibilités allant de l'enseignement en présentiel à l'enseignement à distance en mode totalement asynchrone. Chacun des axes étant un continuum, de nombreuses possibilités pourraient y être ajoutées, notamment l'enseignement comodal sur l'axe 3 ou l'évaluation formatrice sur l'axe 2. Le cube de l'articulation de l'évaluation des apprentissages présente plusieurs possibilités pour évaluer un même objet d'apprentissage, selon les différentes visées de l'évaluation et les divers contextes d'enseignement. II est donc possible d'évaluer l'ensemble des apprentissages de l'élève tant en présentiel qu'à distance. Ainsi, dans le cadre d'un programme de formation où les compétences des élèves sont habituellement évaluées en présentiel, le contexte d'enseignement à distance ne devrait pas pervertir les stratégies d'évaluation déjà en place. Les compétences peuvent donc être évaluées tant en situation d'enseignement en présentiel qu'en situation d'enseignement entièrement à distance. À titre d'exemple, dans un programme visant l'atteinte d'objectifs d'apprentissage, l'évaluation de la maîtrise d'une connaissance (niveau de complexité faible) peut se faire en situation d'évaluation au service de l'apprentissage où l'élève s'autoévalue, et cela, autant en contexte d'enseignement en présentiel qu'en contexte d'enseignement à distance. Pour les programmes centrés sur le développement de compétences, les tâches complexes utilisées pour évaluer le niveau de compétence atteint par l'élève peuvent tout aussi bien être réalisées en contexte d'enseignement en présentiel ou à distance et en situation d'évaluation au service de l'apprentissage ou encore en situation d'évaluation de l'apprentissage. La recrudescence d'évaluations des apprentissages à l'aide d'items à choix multiples ou d'items à réponse courte en situation d'enseignement à distance n'est donc pas justifiée dans des programmes dont la finalité est de développer des compétences, même lorsque les élèves sont à distance ou suivent les enseignements de manière asynchrone. 


\section{Conclusion}

Bien que l'évaluation des compétences à l'aide d'items à choix multiples semble être privilégiée au tournant vers un enseignement à distance, par préoccupation de diminuer le temps de correction ou par l'utilisation de logiciels de surveillance en vue d'une diminution du plagiat, le problème de l'alignement pédagogique demeure bien réel. Sans alignement pédagogique, les informations transmises et les décisions prises à la suite de la réalisation de tâches d'évaluation par l'élève perdent de leur pouvoir informatif et formateur et remettent en cause la pertinence du jugement évaluatif. Quelques solutions peuvent être envisagées pour éviter ces pertes. En voici quelques-unes qui pourraient aussi prévenir le plagiat :

- Donner à l'élève des tâches complexes pour évaluer son niveau de compétence développée quel que soit le contexte d'enseignement.

- S'assurer de l'alignement pédagogique lors de la planification d'évaluation des compétences clôturant des séquences d'enseignement à distance.

- Planifier les activités d'enseignement et les modalités d'évaluation à rebours, soit en partant de ce qui est visé dans le programme de formation pour planifier les modalités d'évaluation des apprentissages et terminer avec la planification des activités d'enseignement.

En bref, le point central de l'évaluation tant en enseignement présentiel qu'en enseignement à distance demeure l'alignement pédagogique. II est tout aussi important de considérer ce qui doit réellement être évalué, et de quelle manière, afin de concevoir des activités d'apprentissages efficaces, comme le mettait déjà en évidence Rieunier (2007). À distance ou en présence, l'alignement, la cohérence pédagogique, reste la clef du succès.

\section{Liste de références}

Baxter, G. P., et R. J. Shavelson. (1994). Science performance assessments: Benchmarks and surrogates. International Journal of Educational Research, 21(3), 279-98. https://doi.org/10.1016/S0883-0355(06)80020-0

Biggs, J. et Tang, C. (2011). Teaching for Quality Learning at University (4e éd.). Mc Graw Hill.

Cao, C. et Qin Q-H. (2015). Hybrid Fundamental Solution Based Finite Element Method: Theory and Applications. Advances in Mathematical Physics 15. http://doi.org/10.1155/2015/916029

Cohen, S.A. (1987). Instructional Alignment: Searching for a Magic Bullet, Educational Researcher, 16(8), 16-20. https://doi.org/10.3102\%2F0013189X016008016

Detroz, P., Tessaro, W. et Younès, N. (2020). Évaluer en temps de pandémie. e-JIREF, (1),1-3. http://journal.admee.org/index.php/ejiref/article/view/212

Durand, M.-J. et Chouinard, R. (2012). L'évaluation des apprentissages: De la planification de la démarche à la communication des résultats. Éditions Hurtubise HMH Ltée.

Earl, L. M. (2013). Assessment as Learning: Using Classroom Assessment to Maximize Student Learning. Corwin Press.

Finson, K. D. (1994). Science Alternative Assessment Models in Illinois. Journal of Science Teacher Education, 5(3), 97-110. https://doi.org/10.1007/BF02614579

Gérard, F.-M. (2009). Évaluer des compétences : guide pratique. De Boeck.

Gremion, C. et Leroux, J. L. (2019). L'évaluation à l'épreuve de la distance et du numérique. Questions vives, 31. https://doi.org/10.4000/questionsvives.3738

Nunziati, G. (1990). Pour construire un dispositif d'évaluation formatrice. Les Cahiers pédagogiques, (280), 47-64. 
Pellegrino, J. W. (2012). Assessment of Science Learning: Living in Interesting Times. Journal of Research in Science Teaching, 49(6), 831-841. https://doi.org/10.1002/tea.21032

Rieunier, A. (2007). Préparer un cours. 2 - Les stratégies pédagogiques efficaces. ESF Éditeur.

Scallon, G. (2004a). L'évaluation des compétences et l'importance du jugement. Pédagogie collégiale, 18(1), 14-20. https://cdc.qc.ca/ped coll/pdf/Scallon 18 1.pdf

Scallon, G. (2004b). L'évaluation des apprentissages dans une approche par compétences. Éditions du Renouveau pédagogique.

Tyler, R. W. (1949). Basic Principles of Curriculum and Instruction. The University of Chicago Press.

Vial, M. (2012). Se repérer dans les modèles de l'évaluation. Méthodes, dispositifs, outils. De Boeck Université. 